\title{
Hipertensión renovascular en pediatría
}

\author{
Dr. Carlos Saieh A.; Di. Victor Diaz B.; ${ }^{2}$ Dr. Francisco Valdés E.: ${ }^{3}$ \\ Dra. Katica Ramirez P.4; Dr. Jose D. Arce V. 2 \\ Renoyascular hypertension
}

\begin{abstract}
Among 122 hypertensive children seen jrom 1976 to 1986 in a pediatric hospital at Santiago, Chilc, evidener of a renovasculat cause was detected in ten. Their mean age at presentition was 6.2 years (tange 2 - 149 montlrs); the most frequent complaint was headache; mean systolic and diastolic pressurts were 168 and $113.5 \mathrm{~mm} \mathrm{Hg}$ respectively. Renal function was normai. Intravenous pyelography dikclosed abnomalities in seven cases. Artsrial angiography showed evidence of Takavasu's artwritis in 4 pationts and jibromuscular dysplasia ir 2. In threc cases complete oclussinu of a renal artery was found: howcver only onc had renal perfusion through cotateral circulation wich allowed surgical revascularization. On: cass of renal stcnosis was succenlully treated by percutaneous transluminal angsioplasty. Selwctive medicaj treatment. specially in active Takayasu's disease, appropriate surgical correction or translummal dilatation resulted in cure of hypertinsion in this series.
\end{abstract}

(Key words: arterial hypertension, renovascular, medical and surgical treatment)

Actualmente se reconoce que la hipertensión arterial es una importante causa de morbimortalidad, que afecta a personas de todas las edades; ${ }^{1.2}$ su prevalencia en adultos es aproximadamente $20 \% ;{ }^{3}$ oscilando entre 0,6 y $11,0 \%$ en niños ${ }^{4}$

En pediatría, la hipertensión secundaria ocupa un lugar preferente; en nuestra experiencia $82.6 \%$ de los pacientes con hipertensión arterial corresponden a una causa renal y de ellos $9 \%$ tienen origen renovascular. ${ }^{5}$ Experiencias extranjeras han demostrado que las reparaciones de las lesiones arteriales responsables do la hipertensión renovascular (HRV) tienen una evolución muy satisfactoria, abrigando esperanzas de un futuro mejor para estos pacientes de corta edad. ${ }^{6 \cdot 10}$

Presentamos una experiencia en el manejo de la HRV en una unidad de nefrourologia analizando fundamentalmente los metodos de estudio, el tratamiento y la cvolución inmediata, según las diferentes etiologias, con el objeto de dar a conocer un problema, que aunque poco frecuerte, requiere especial consideración para su diagnóstico precoz y tratamiento oportuno.

1. Unidad de nefrourología, Hospital Luis Cako Mackenna. Departamento di Pediatría. División Clencias Médicas Oriente, Facultad de Medicina Universidad de Chile.

2. Servicio de Radiologia, Hospital Luis Calwo Mackenna.

3. Departamento de enfermedades cardiovasculares, Cirugía vascular, Liscuela de Medicina y Hospital Clínico. Pontificia Universidad Catolica de Chile

4. Becaria de Ptdiatría, Servicio de pediatria, Hospital Luis Calvo Mackinna.

\section{MATIRIAL Y METODOS}

Entre 1970 y 1986, sc han controlado 122 niños hipertenson en la unidad de nefrourología del Hospital Lujs Calvo Mackenna. En 10 de ellos se diagnósticó hipertemsión de otigen renovaseular (tabla). En todos clios se realizaron radiogralias de lorax, electrocardioerumra, pielografia minutada, atterogratía renal y estudios de depuración de creatinina. Sólo en dos pacientes lute posible hacer mediciones de renina plasmátía. Los antecedences clínicos y de labotatorio fueron Jevisados mediante un protocolo retrospectivo.

\section{RESULTADOS}

En ul momento de la primera consulta, la edad promedio fue 6, 2 años (rango 2 meses a 12 tนños 5 meses), siendo 4 varones y 6 niñas. El motivo de consulta más frecuente fue cefalea $(60 \%)$, de predominio matinal en Ia mayoría de los casos: más raramente $(10$ a $20 \%$ ) hubo fiebre, convulsión, irritabilidad, dolor abdominal y hematuria.

La presión sistólica promedio fue de $168 \mathrm{~mm} \mathrm{Hg}$ (rango 110 a 210 ) y la diastólica pronedio fue $113.5 \mathrm{~mm} \mathrm{Hg}$ (rango 85 a 140). El fondo de ojo reveló edema leve y constricción arteriolar en 3 pacientes mayores de 6 años. alteración que fue transitoria, desapareciendo en pocas sejnanas. Sólo en dos casos se auscultaron suplos abdominales, uno tenia arteritis de Takayasu y otro estenosis de la arteria renal.

Ocho niños presentaron crecimiento ventricular izquierdo tanto al ECG como en la radiografía de tórax. Siete de ellos mostraron en la pielografía alguna altcración sospechosa de hipertensión renovascular, fundamentalmente 
Tabla

Hechos clínicos más destacados en 10 niroos con hipertensión arterial renovascular

\begin{tabular}{|c|c|c|c|c|c|c|c|}
\hline Paciente & $\begin{array}{c}\text { Edad } \\
\text { (Años, neves) }\end{array}$ & $\operatorname{SexO}$ & Dją̧nóstico & $\begin{array}{l}\text { Presión arnerial } \\
\text { Pre-tratamiento } \\
\left.\text { (गm } \mathrm{Hg}_{\mathrm{g}}\right)\end{array}$ & Ttatamicn to & $\begin{array}{l}\text { Presión arterial } \\
\text { Pos-trataraiento } \\
\text { (mm Hg) }\end{array}$ & Evolución y seguimiento \\
\hline L) M.C.B. & $6 a$ & $M$ & $\begin{array}{l}\text { Fislenosis arteria } \\
\text { renal izquierda }\end{array}$ & $185 / 125$ & Het $\mathrm{P}-\mathrm{H}$ & $140 / 110$ & $\begin{array}{l}\text { Fatlecido por compromiso } \\
\text { cardiovascular. } 1 \mathrm{a} 2 \mathrm{~m}\end{array}$ \\
\hline 2) E.C. & $6 \mathrm{a} 6 \mathrm{~m}$ & $F$ & $\begin{array}{l}\text { Oelusión at retia } \\
\text { renal izquie rda }\end{array}$ & $155 / 105$ & $\begin{array}{l}\text { By.Pass aarto } \\
\text { cenal izquierda }\end{array}$ & $130 / 60$ & la $2 m$ \\
\hline 3) Y.B. & $6 \mathrm{am}$ & $\mathrm{I}:$ & $\begin{array}{l}\text { Aneurisona arterid } \\
\text { end] isquic rdid }\end{array}$ & $\mathrm{IBO} / 120$ & $\begin{array}{l}\text { Extirpacion ancu- } \\
\text { risma Hct. P.H. }\end{array}$ & $150 / 100$ & $6 a 7 m$ \\
\hline 4) J.A.P. & $2 \pi$ & $\mathbf{M}$ & $\begin{array}{l}\text { Trombusis alterla } \\
\text { renal lźquicrda }\end{array}$ & $140 ; 95$ & Hct. P. & $120 \div 70$ & Exçlusión renal. 2a 4m \\
\hline 5) A.B. & $3 m$ & $M$ & $\begin{array}{l}\text { Ojsplasia fibromu veu. } \\
\text { lar aft renal jzquietda }\end{array}$ & $160 / 110$ & Het. P. & 110,60 & Exclusión renal. 6a $2 \mathrm{~m}$ \\
\hline б) $\mathrm{MJ}$ & $11 d 7 \mathrm{mo}$ & F & $\begin{array}{l}\text { Displasia fibromuscu- } \\
\text { lat art. ronal izquierda }\end{array}$ & $160 / 110$ & $\begin{array}{l}\text { Angioplastía per- } \\
\text { cutánea translu. } \\
\text { minat }\end{array}$ & $110 / 70$ & 10 meses \\
\hline 7) D L. & $9 \mathrm{a}$ & $1:$ & Anle ritis Takayasu & $170: 100$ & HLt - P.-H - Pr. & $150 / 100$ & $\begin{array}{l}\text { Fallccida por septicemia } \\
\text { la } 2 \mathrm{~m}\end{array}$ \\
\hline B) M.S. & $6.7 \mathrm{~m}$ & $\mathrm{M}$ & Ar1:xid Takayasu & $210 / 140$ & $\mathrm{Hct}-\mathrm{P} \mathrm{Min}-\mathrm{Pt}$ & $130 / 80$ & $3 a 2 \mathrm{~m}$ \\
\hline 9) M.J.G. & int & $\boldsymbol{r}$ & Artentis Takayasu & $160 ; 120$ & $\mathrm{Het}-\mathrm{P}-\mathrm{H}-\mathrm{P}_{\mathrm{t}}$ & 130,75 & $4 \mathrm{a} 4 \mathrm{~m}$ \\
\hline 10) M.F. & $12 \mathbf{a} 5 \pi$ & 1 & Arteriti Takayasiu & $160 ; 110$ & Het $\cdot \mathrm{P}-\mathrm{PI}$ & $150 ! 100$ & $\begin{array}{l}\text { Fallecida por Pertonitis } \\
\text { fecaloidea. } 6 \mathrm{~m}\end{array}$ \\
\hline
\end{tabular}

Hęt $=$ Hidrosloro tiaz.ida

$\mathrm{P}=$ Propranolo]

H $=$ Hidralacina

Pt $=$ Prednisona

Min $=$ Minoxid山

retardo de eliminación del medio de contraste en el riñón isquémico, y menor tamaño en comparación con el contralateral. Actividad de renina plasmática se midió sólo en 2 niños por falta de facilidad para efectuarla en condiciones óptimas en el momento oportuno.

La arteriografía permitió el diagnóstico de arteritis de Takayasu en 4 casos (figura l). Por considerarse en fase activa, fucton tratados con prednisona, diuréticos y asociación de propanolol $y$ vasodilatadores. Dos niños fallecieron a los $3 y$ 14 meses. a consecuencia de cuadros septicos complicados; la necropsia se realizó sólo en uno de ellos, confirmándose el diagnóstico. Los dos sobrevivientes mantienen cifras tensionales normales y desarrollo pondoestatural adecuado para su edad, Juego de 38 y 52 meses de control.

En un caso en que se diagnosticó aneurisma de arteria renal, éste fue reparado quirúrgicamente, siendo necesario igualmente mantener el tratamiento para obtener la normotensión.

En los cinco pacientes restantes se demostró compromiso oclusivo severo de arteria renal de predominio unilateral. Angiográficamente las lesiones fueron de tipo displasia fibrosa. En tres la oclusión arterial era completa, con atrofia renal en dos, y ent el tercero, Ja perfusión renal se hacía por colaterales (figura 2), lo que permitió irrigar el riñón mediante un puente vascular aor to renal (figura 3), curando la hipertensión.

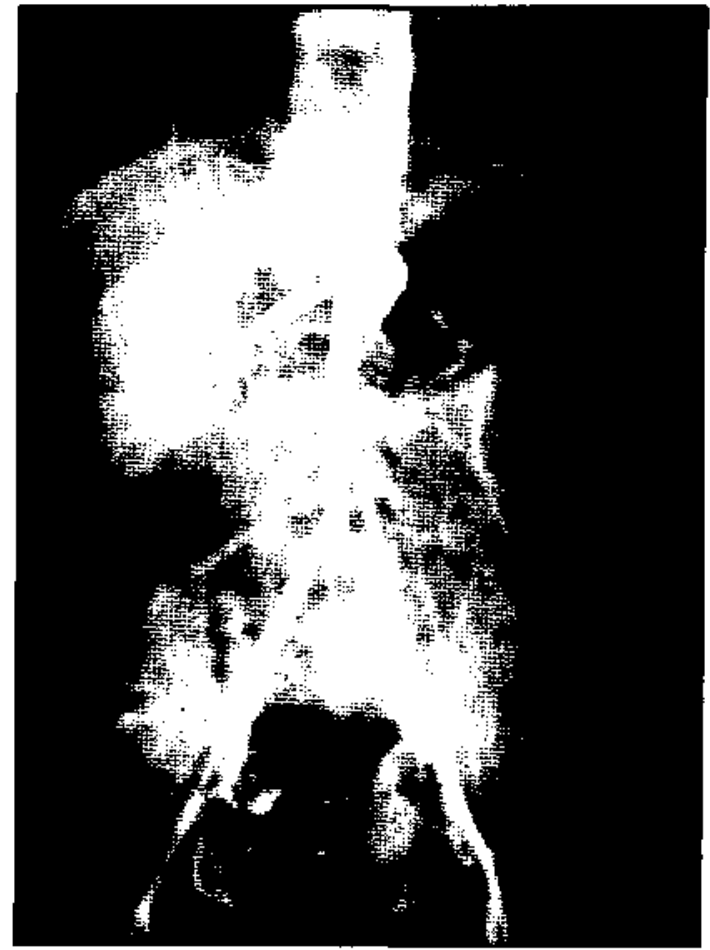

Fugura 1: Aortografía. Imágenes sugerentes de arteritis de Takayasu. La afteria aorta muestra irregularidades y reducción de su calibre; en la arteria mesentérica existe una dilatación. Se observa una estenosis filiforme de la arteria renal derecha y oclusión completa de la izquierda. 


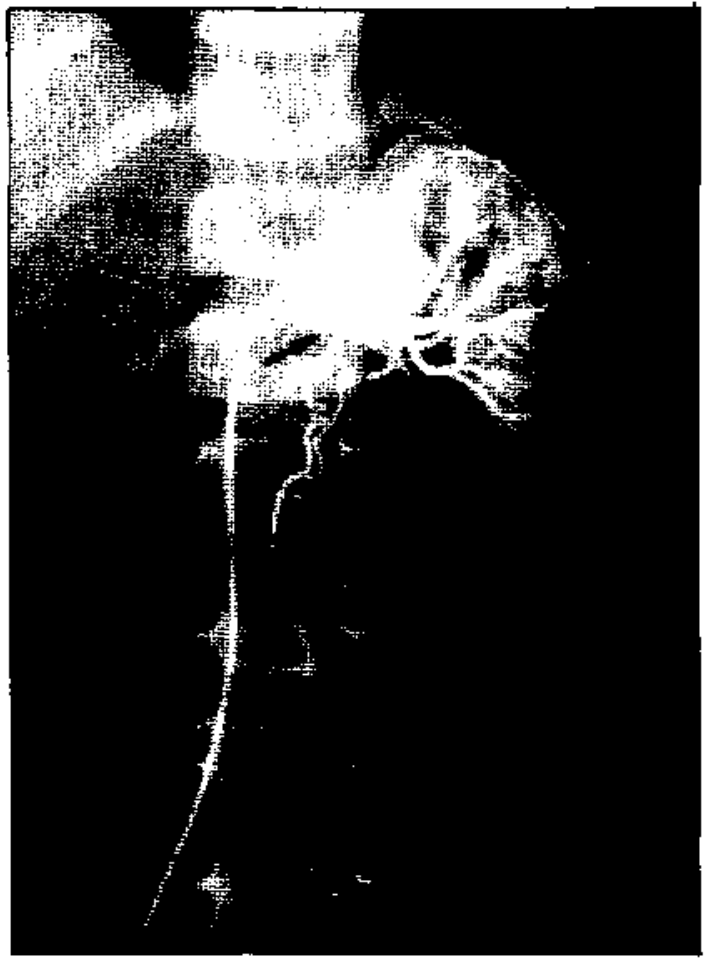

Figura 2: Arteriografía. Oclusión completa en el nacimiento de la arteria renal izquierda, que se ltena a través de colaterales.

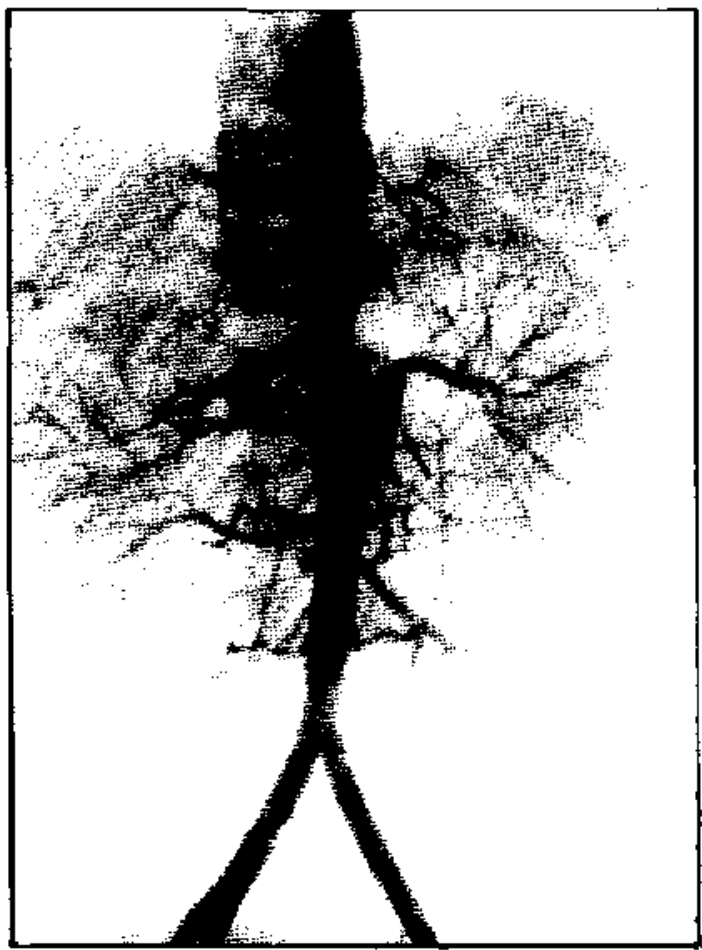

Figura 3: Arteriografia. Puente vascular aorlo-jenal izquic rdo con vena safena. Control 12 mescs después de la reparación quirútgica.
Los dos niños restantes presentaban estenosis renal. Uno falleció por causas cardiacas, debido a que era portador de ventrículo único, inoperable y no compatible con la vida en edad más avanzada; el otro fue sometido exitosamente a dilatación transluminal percutánea con desaparición de la hipertensión y en buenas condiciones hasta 10 meses de control después del procedimiento.

\section{DISCUSION}

La hipertensión arterial en níños ha despertado el interés de los pediatras en forma progresiva. Aunque la incidencia de la HRV es baja, ella es potencialmente curable, lo que obliga a buscar esta causa. Diversas publicaciones han establecido las características clínicas relevantes de la HRV, así como el rendimiento de los diferentes métodos de diagnóstico y las expectativas de los recursos terapeuticos disponibles en la actualidad. ${ }^{10-19}$

La ausencia de sintomas y signos clínicos especificos en las etapas iniciales de la hipertensión hace importante incluir el control de la presión arterial en el examen físico de rutina. En aquellos casos de mayor severidad o larga evolución sueie ser evidente la irritabilidad, curva pondoestatural mantenida, cefalea. vómitos, etc. ${ }^{20}$ En esta pequeña serie el $60 \%$ de los pacientes acusaba cefalea de predominio matinal $y$ en dos casos fue manifiesto un sindrome convulsivo.

Considerando el alto indice de curabilidad de la HRV en niños, ${ }^{17}$ y por otra parte el daño progresivo multisistémico a que puede llevar, en caso de no ser diagnosticada en etapas precoces, el estudio de la hipertensión arterial debe ser agresivo, cubriendo todo el espectro de exámenes disponibles. ${ }^{21,22}$

E1 hallazgo de soplos abdominales, anomalías de los pulsos, presencia de manchas "café con leche", hipertrofia ventricular izquierda, etc., orientan hacia el diagnóstico de HRV. Las alteraciones del fondo de ojo suelen ser leves, encontrando edema papilar o algún grado de contricción arteriolar con poca frecuencia. En 3 de nuestros pacientes, todos mayores de 6 áños, se encontraron alteraciones of talmológicas, lo que lace suponer que es necesario un período de latencia prolongado para alcanzar el daño retiniano $\tan$ frecuente en adultos con hipertensión secundaria.

La pielografía de eliminación orienta el diagnóstico si se demuestra retardo de eliminación del contraste en el rînón isquémico, asimetría en lis longitud renal, hiperconcentración del contraste en el riñón afectado y/o, indentaciones en el sistema pieloureteral determinadas por la com- 
presión de red colateral hipertrófica, En 7 de nuestros casos hubo algún elemento orientador, sin embargo, dado que las lesiones vasculares son bilaterales en más de $25 \%$ de los niños, el rendimiento diagnóstico de la pielografía puede ser limitado. 17,23 Aunque la pielografía resulte normal, si se sospecha HRV, se debe recurrir a la arteriografia, único examen que demuestra las lesiones en forma precisa. La angiografía por sustracción digital, en que el contraste es intensificado mediante computadores y requiere dosis minimas de medio de contraste, si es empleado por vía intraarterial, o evita la instrumentación arterial directa si se inyecta en una vía venosa, ha significado ahorro en costo y riesgos del estudio, pudiendo efectuarse en forma ambulatoria. ${ }^{24} \mathrm{Sin}$ embargo, los artefactos pueden ser más frecuentes que en el estudio clásico, đebido a movimientos $o$ incluso alteraciones del débito cardíaco, restando resolución al estudio. 25

La repercusión de la estenosis arterial sobre la función renal global sólo se manifiesta en los casos de curso agudo y habitualmente bilateral dada la elevada reserva funcional del niño y su capacidad de desarrollo de circulación colateral, lo que explica que en los 10 pacientes la depuración de creatinina fue normal.

Nuestra experiencia con la actividad de renina plásmatica es muy reducida por no disponer de los medios materiales para realizar este examen en el momento adecuado. Además, todos los pacientes recibían terapia antihipertensiva, siendo imposible descontinuarla pot sus altas cifras tensionales. La actividad de renina plasmática está afectada por muy diversos factores y su valor en la decisión terapéutica es discutido, ${ }^{26,27}$ razón por la cual no ha sido considerada por nosotros como un examen fundamental en el estudio de los niños con HRV

Con la excepción de los casos secundarios a arteritis de Takayasu en fase activa, confirmado el diagnóstico de hipertensión de causa renovascular, el tratamiento más efectivo es la intervención sobre la arteria estenótica u oclúsda. ${ }^{11,17,19,26}$ Durante la actividad inflamatoria en la enfermedad de Takayasu se ha demostrado recuperación funcional renal con tratamiento cortjcoesteroidal, ${ }^{27}$ siendo posible postergar una eventual cirugia en aquellos casos que ta oclusión vascular evoluciona a la cronicidad en la fase resolutiva. En los casos de arteritis de nuestra serie que sobreviven, el tratamiento médico ha permitido un desarrollo pondoestatural adecuado, sin compromiso de la función renal y normotensión, a 3 y 4 años plazo del inicio de la terapia.

El tratamiento quirúrgico en series controladas hasta un promedio de 42 meses, ha mostrado curación en $85 \%$ y mejoría en $12,5 \%$ de los casos. ${ }^{26}$ La técnica de elección es el puente aorto renal con material autólogo (arteria hipogástrica o vena safena), reservándose el autotransplante para lesiones complejas segmentarias o del hilio renal. La nefrectomía sólo está indicada en lesiones segmentarias irreparables (nefrectomía parcial) o casos de exclusión y atrofia renal que se acompañan de hipertensión de difícil manejo médico. De esta reducida șerie, 3 niños se presentaron con obstrucción renal completa, uno de ellos con riñon funcionante por colaterales, lo que permitió el rescate de dicho órgano con un puente aorto-renal lográndose la curación de la hipertensión (figuras 2 y 3 ).

El rol de la dilatación transluminal percutánea va adquiriendo una importancia mayor, ya que a un menor riesgo y costo, logra la recuperación de la estenosis en los casos de displasia fibrosa, causa frecuente en niños, que puede ser de rápida evolución llegando a la oclusión completa, ${ }^{24,25}$ Los resultados de la angioplastia en el largo plazo aún están en evaluación, sin embargo, en los casos de arteritis de Takayasu, dicha terapia debe ser desaconsejada por el riesgo de reestenosis inflamatoria $y$ oclusión precoz, con eventual pérdida renal.

Los excelentes resultados obtenidos con las medidas terapéuticas actuales en el tratamiento de la hipertensión renovascular en el niffo justifican ampliamente todos los esfuerzos en investigar intensamente la etiología de la hipertensión en este grupo etario. Así también y aunque nuestro grupo de pacientes es reducido creemos que la renoyascularización con preservación del parénquima renal, en los casos aconsejables, debiera ser la meta fundamental en la hipertensión renovascular, exceptuando la arteritis de Takayasu en donde el tratamiento médico ha dado tan buenos resultados.

\section{RESUMEN}

Se presentan 10 niños con hipertensión renovascular controlados en el Hospital Luis Calvo Mackenna desde 1976 a 1986, de entre una serie de 122 pacientes con hipertensión observados en el mismo periodo. Cuatro etan varones y 6 mujeres y sus edades fluctuaron entre 2 meses y 12 años 5 meses. El principal motivo de consulta fue cefalea $(60 \%)$ de predominio vespertino. Siete de los 10 pacientes mostraron alteración saspechosa de hipertensión renovascular en la pielografía y en todos ellos se confirmó el diagnóstico por arteriografía. En 4 nifros se diagnóstico arteritis de Takayasu, en 2 displasia fibromuscular y en cada uno de los restantes trombosis, aneurisma, oclusión y estenosis de la 
arteria renal. Se analizan los tratamientos en cada una de las patologías, haciendo hincapie en los buenos resultados obtenidos con la cirugia y la angioplastia transluminal percutánea, asi como con el tratamiento médico en la arteritis de Takayasu. Sea cual sea el tratamiento la finalidad es preservar el parénquima renal y la terapia a usar es individual para cada paciente.

\section{REFERENCIAS}

1. Adams F.H., Landau E.M.: What aro healthy blood pressures for children? Pediatrics 1981; 68: $268-270$.

2. I.oggie J.M.H., Horan N.J., Hohn A.R., Gruskin A.B., Dumber J.B., Hovlik R.J.: Juvenile hyperten. sinn: highlight of a workshop. I Podiatr 1984; 104 $657-663$.

3. Inter-Society Commission for heart disease. Resources, guidulines for the detection, diagnosis and management of hypertensive populations. Repurt of the hypertension study group. Circula tion $1971 ; 44:$ A $263-286$

4. Gyarfas I.: Blood pressure in childhood and adolescence. Result from an interrational collaboration study on juvenile hypertension. Acta Pediatr Scand (Suppl) 1985;313:11-12.

5. Saieh C., Ramirez K.: Hipertensión renovascular en niños. XI Jornadas Chilenas de Pediatrít. Santiago-Chile, Noviembre 1985 .

6. Wooddruff M.F. Doig A., Donald K.W., Nolen B. Renal aulotrasplantation Lance $1966 ; 1 ; \mathbf{4 3 3 - 4 3 6 .}$

7. Chunic G.J.A., Gordon R.D., Hartley L.C.J.: Results of surgical treatment of renovasculas hypertensión. Med J Aust 1975;1: 225.230.

8. Kuhlmann $U$., Vetter $\boldsymbol{w}$, Fumer J., Lutolf $U$., Sicgenthater W. Gruntzin A.: Renovastular hypertension. Treatment by percutaneous trasluminal dilatation. Ann Intern Med 1980; $92: 1-6$.

9. MC Cook T.A., Mills S.R., Kirks D.R., Heaston D.K., Siegler H.F., Malone R.B., Osofky' S.G.: Percutancous trasluminal renal artery angioplasty in a $31 / 2$ year old hypertensive giri. J Pediatr 1980; 97: $958-960$

10. Watson A.R. Balfe W., Hardy B.E.: Ranovascular hypertension in childhood a changing perspective in management. J Pediatr 1985; $106: 366-372$.

11. Novick A.C., Straffon R.A., Stewart B.H., Benjom in $S$, : Surgical treatment of renovascular hypertension in the pediatric patient. $J$ Urol 1978; 119: 794-798.

12. Malin S.W., Baumsart S., Rosenberg H., Foreman $J$.: Nonsurgical managennent of obstructive aortic thrombosts complicated by renovascular hyperten- sion in the neonate. I Pediatr 1985; 106:630-634.

13. Barber W.F.: Current validity studies of blood pressure instrumentation. Hypertension $1984 ; 6$ : 85-89.

14. Lawson J., Boerth R., Foster J.H., Dean R.H. Diagnosis and managejnent of renovascular hypertension in children Arch Surg 1977; 112: $1307-1316$.

15. Awazu M. Shimizu M., Hojo H., Osano M., Kohdo E.: Percutaneous transluminal angioplasty for renovascular hypertension. Arch Dis Child 1985; 60: 627-630.

16. Barth K.H., Brusilow S.W., Kaufman S.L., Ferry F.T.: Percutancous transluminat angioplasty of homograft renal artery stenosis in a 10 year old girl. Pediatrics 1981;67:675-677.

17. Stanley I.C., Fry W.J.: Pediatric renal artery occlusive disease and renovaseular hypertension. Arch Surg 1981; 116:669-676.

18. Hendren H.W., Kim S.H., Herrin J.T., Crawford J.D.: Surgically correctable hypertension of renal origin in childhood. Am J Surg 1982; 143: 432.442.

19. Reintgen D., Wolfe W.G, Osofky S., Seigler H.T.: Artery stenosis in children. J Pediatr Surg 1981; 16: 26-32.

20. Makker S.P. Lubonhn S.D.: Clinical features of renovascular hypertension in infancy. Report of a 9 month old infant. Pediatrics 1975: 56: 108-110.

$2 \mathrm{~J}$. Londe $S:$ : Causes of hypertension in the young. Pediat Clin North An 1978; 25: 55-65

22. Lawson J.D., Boarth R., Foster J.H.: Diagnosis and management of renovascular hypertension in chitdren. Asch Surg 1977: 112:307-314.

23. Stantey P., Gyepes M.T. Olson D.L., Gates G.F.: Renovascular hypestension in children and adolescents. Radiology 1978; 129: $123 \cdot 129$.

24. Mistretto C., Crumy A., Strother C.M.: Digital angiography: A perspective. Radiology 1981; 139: 273-278.

25. Capizaneo M.A., Faerber E.N., Gainey M.A., Wolfson $B . J .:$ Digital sustraction angiography and its application in children. Pediatr Clin Sorth Am $1985 ; 32: 1449-1460$

26. Sawson J.D., Boerth R., Foster J.H., Dean R.H.: Diagnosis and management of renovascular hypertension in children. Arch SuIg 1977; 112: 1303-1314

27. Lagneau P., Michel J.B.: Renoyascular hypertension and Takayasu's disease. J Urol 1985; $134: 8$ ?6-879.

28. Stanley J.C.: Runal vascular disease and renovascu. lat hypertension in children. Urol Clin North Am $19.84 ; 1]: 451-463$.

29. Saieh C., Yurlow E., Arce J.D. y col.: Arteritis de Takayasu en una lactante. Bol Méd Hosp Inf México, $1985 ; 42: 135-139$ 